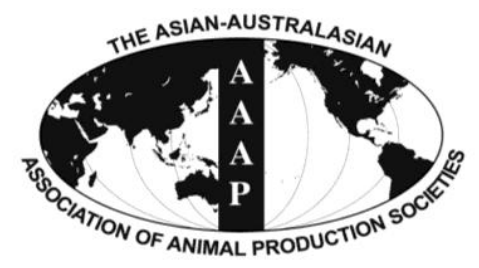

Asian-Aust. J. Anim. Sci.

Vol. 25, No. 5 : 629 - 634

May 2012

www.ajas.info

http://dx.doi.org/10.5713/ajas.2010.10249

\title{
Growth of Ovarian Primary Follicles Retrieved from Neonates of Different Ages and Derivation of Mature Oocytes Following In vitro-Culture
}

\author{
J. H. Choi ${ }^{1}$, C. R. Yoo ${ }^{1}$, J. Y. Ahn' ${ }^{2}$, J. H. Park ${ }^{1}$ and J. M. Lim ${ }^{1,2,3, *}$ \\ ${ }^{1}$ Department of Agricultural Biotechnology, Seoul National University, Seoul 151-912, Korea \\ ${ }^{2}$ WCU Biomodulation Program, Seoul National University, Seoul 151-742, Korea \\ ${ }^{3}$ Institutes for Agriculture and Life Sciences, Seoul National University, Seoul 151-921, Korea
}

\begin{abstract}
This study was conducted to improve the yield of mature oocytes from in vitro-culture of ovarian primary follicles by optimizing follicle retrieval from neonatal mice of different ages. Primary follicles of 75 to $99 \mu \mathrm{m}$ in diameter were collected daily from 7- to 14-day-old neonatal mice, and subsequently cultured in $\alpha$-MEM medium. Number of primary follicles isolated, growth of the follicle during in vitro-culture and maturation of intrafollicular oocytes were monitored. Overall, mean number of preantral follicles per animal was improved from 10.7 to 88.7 as the age of follicle donors was increased from 7 to 14-day-old. Number of primary follicles was increased gradually up to 11-day-old (35.7 follicle per an animal), then reduced to 29 in 14-day-old ( $p=0.0013)$. More follicles retrieved from 10-day-old or 11-day-old females maintained their morphological normality at the end of primary culture than the follicles retrieved from 9-day-old. Of those cultured, primary follicles retrieved from 11-day-old mice yielded largest larger number of early secondary follicles than the follicles retrieved from in the other ages (39 vs. 13 to $29 \%$ ). More than 3.3-times increase (0.86 to 2.86; $\mathrm{p}<0.05)$ in an average number of mature oocytes per animal was observed in the group of 11-day-old, compared with 9-day-old. However, no difference was found in the percentage of primary follicles developing into the pseudoantral stage $(21$ to $30 \%$; $\mathrm{p}=0.5222)$ and in the percentage of oocytes mucified ( 32 to $39 \% ; \mathrm{p}=0.5792$ ). In conclusion, a positive correlation between retrieval time and follicle growth was detected, which influences the efficiency to derive mature oocytes by follicle culture. (Key Words: Neonatal Mouse, Ovary, Primary Follicle, In vitro Culture, Oocyte Maturation)
\end{abstract}

\section{INTRODUCTION}

We have made an attempt to developing efficient preantral follicle culture system for generating developmentally competent oocytes, and several culture strategies for secondary follicles have been developed to date (Lee et al., 2007; Lee et al., 2008; Kim et al., 2009). This culture system has been employed neonatal female mice as the follicle donor and has served a recent success on developing of follicles derived from adult ovaries (Kim et al., 2009). Viable blastocysts has also been generated from IVF or parthenogenetic activation of the folliclederived oocytes (Gong et al., 2008; Lee et al., 2008; Gong et al., 2009; Kim et al., 2009), which confirms the feasibility of the follicle culture system in reproductive

\footnotetext{
* Corresponding Author: Jeong Mook Lim. Tel: +82-2-880-4806, Fax: +82-2-874-2555, E-mail: limjm@snu.ac.kr Submitted Jul. 6, 2010; Accepted Aug. 31, 2010: Revised Dec. 31, 2010
}

biotechnology and stem cell engineering. However, most follicle culture techniques deal with secondary follicles and a very limited success on the culture of primary follicles has been made to date (Lenie et al., 2004). These primary follicles are the most abundant among the growing pool of ovarian follicles (O'Brien et al., 2003). Thus, their successful mobilization for generating developmentally competent oocytes can greatly increase feasibility of preantral follicle culture system.

In this study, we attempted to further optimize a primary follicle culture system by evaluating of appropriate retrieval time. The follicles retrieved at different times from neonatal females were cultured for obtaining mature oocytes, and assessment on the efficiency of follicle retrieval and mature oocyte generation was subsequently made by employing both cellular and biological parameters. Prospective randomized trial was conducted with using of F1 hybrid mice. 


\section{MATERIAL AND METHODS}

\section{Experimental design}

We first evaluated an appropriate time to retrieve primary follicles from neonatal ovaries (experiment 1 ). The numbers of primary, and early and late secondary follicles retrieved from prepubertal ovaries of 7- to 14-day-old females were monitored and changes in the number of primary follicles were concomitantly evaluated. In experiment 2 , primary follicles retrieved from optimal ages of retrieval (9- to 11-day old mice) were cultured for 11 days. Morphological integrity and growth to the pseudoantral follicles, oocyte mucification, and initiation (germinal vesicle breakdown) and completion (metaphase II plate formation) of meiotic maturation were subsequently monitored.

\section{Experimental animal}

As an experimental animal, B6CBAF1 (C57BL6x $\mathrm{CBA} / \mathrm{ca}$ ) mice were employed. All procedures for animal management, breeding, and surgery followed the standard protocols of Seoul National University, Korea. The Institutional Animal Care and Use Committee at Seoul National University approved our research proposal in April 2005 (SNU0050331-02).

\section{Isolation of preantral follicles}

The ovaries retrieved from euthanized females were placed in L-15 Leibovitz-glutamax medium (Gibco Invitrogen, Grand Island, NY) supplemented with $10 \%$ (v/v) heat-inactivated fetal bovine serum (FBS; HyClone Laboratories, Logan, UT). Preantral follicles were mechanically isolated from neonatal mice by using a 30gauge needle without enzymatic digestion (Nayudu et al., 1992; Lenie et al., 2004). After retrieval, the follicles were classified according to their size and morphology (Smitz et al., 2002; Lee et al., 2008). Primary follicles selected were placed singly in $10 \mu$ l culture droplets overlaid with mineral oil in a $60 \times 15 \mathrm{~mm}$ culture dish.

\section{In vitro culture and in vitro maturation}

The medium used for the culture of primary follicles is a ribonucleoside and deoxyribonucleoside-free, $\alpha$-MEMglutamax medium (primary culture medium), to which $1 \%$ (v/v) heat-inactivated FBS, $1 \%$ insulin, transferrin and selenium liquid medium (ITS; Sigma-Aldrich Corp.), 200 mIU recombinant FSH (Organon, Oss, The Netherlands), $10 \mathrm{mIU} / \mathrm{ml} \mathrm{LH} \mathrm{(Sigma-Aldrich} \mathrm{Corp.)} \mathrm{and} \mathrm{1 \%} \mathrm{(v/v)}$ penicillin/streptomycin were added. On day 1 of culture, an additional $10 \mu \mathrm{l}$ fresh medium was added to each droplet and half of the culture medium was changed with fresh medium on day 3 . Then, morphological normality and the size of primary follicles were monitored under an inverted microscope at the end of primary culture (day 4).

Only the follicles with normal morphology were further cultured in $10 \mu \mathrm{l}$ droplets of a ribonucleoside and deoxyribonucleoside-containing $\alpha$-MEM-glutamax medium (Gibco Invitrogen.) supplemented with 5\% heat-inactivated FBS, $1 \%$ ITS solution, $100 \mathrm{mIU} / \mathrm{ml} \mathrm{FSH}$ and the antibiotics (secondary culture medium) from day 4 of culture. On day $5,10 \mu \mathrm{l}$ of fresh medium was added to culture medium and half of the medium was changed daily with fresh medium from day 7 to the end of secondary culture (day 11). To trigger maturation, $2.5 \mathrm{IU} / \mathrm{ml}$ hCG (Pregnyl $^{\mathrm{TM}}$; Organon) and $5 \mathrm{ng} / \mathrm{ml}$ epidermal growth factor (EGF; Sigma-Aldrich Corp.) was added to culture medium $16 \mathrm{~h}$ prior to the end of the culture.

\section{Monitoring of follicular growth and oocyte maturation}

The number of the follicles formed pseudo-antrum, an intercellular space between granulosa cells in cumulusoocyte-complex was counted on day 11 of culture. The number of oocytes spontaneously dissociated from granulosa cell mass (defined as mucified oocytes) was evaluated on day 12 of culture. Subsequently, oocytes retrieved at the end of the second phase culture were freed from cumulus cells by mechanical pipetting and subsequently placed in M2 medium (Lee et al., 2008) supplemented with $4 \mathrm{mg} / \mathrm{ml}$ BSA and $200 \mathrm{IU} / \mathrm{ml}$ hyaluronidase. A comparison of the development of intrafollicular oocytes into the germinal vesicle breakdown (represent as the initiation of maturation) and metaphase II stage (represent as the completion of meiotic maturation) was made between the ages of retrieval. Meiotic maturation of the oocytes was determined by the expansion of cumulus cells and the presence of a first polar body in the perivitelline space.

\section{Statistical analysis}

Numerical data obtained from a randomized, controlled trial were statistically analyzed with a generalized linear model (PROC-GLM) in a Statistical Analysis System (SAS) program. When significant model effect was detected, each treatment effect was compared by the least square method. The level of significance in model effect and pair comparison was determined when $\mathrm{p}$ value was less than 0.05 .

\section{RESULTS}

\section{Experiment 1: Number of preantral follicles retrieved from prepubertal females}

Overall, mean number of preantral (primary, early secondary and late secondary) follicles per neonatal females 
Table 1. Number of preantral (primary, early secondary and late secondary) follicles retrieved from prepubertal mice of different ages

\begin{tabular}{|c|c|c|c|c|}
\hline \multirow[b]{2}{*}{ Ages (days) } & \multirow{2}{*}{$\begin{array}{c}\text { Mean no. }( \pm \mathrm{SD}) \text { of follicles } \\
\text { retrieved (total) }\end{array}$} & \multicolumn{3}{|c|}{ Mean no. $( \pm \mathrm{SD})$ of retrieved follicles at the stage of } \\
\hline & & $\begin{array}{c}\text { Primary } \\
(75-99 \mu \mathrm{m})\end{array}$ & $\begin{array}{l}\text { Early secondary } \\
(100-125 \mu \mathrm{m})\end{array}$ & $\begin{array}{l}\text { Late secondary } \\
\qquad(<126 \mu \mathrm{m})\end{array}$ \\
\hline 7 & $10.7 \pm 8.1^{\mathrm{a}}$ & $8.7 \pm 7.0^{\mathrm{a}}$ & $2.0 \pm 2.0^{\mathrm{a}}$ & $0.0 \pm 0.0^{\mathrm{a}}$ \\
\hline 8 & $36.3 \pm 23.2^{\mathrm{ab}}$ & $18.0 \pm 9.6^{\mathrm{ab}}$ & $17.0 \pm 11.5^{\mathrm{ab}}$ & $1.3 \pm 2.3^{\mathrm{ab}}$ \\
\hline 9 & $53.7 \pm 5.1^{\mathrm{bc}}$ & $31.0 \pm 3.6^{\mathrm{c}}$ & $18.3 \pm 2.1^{\mathrm{ab}}$ & $4.3 \pm 4.5^{\mathrm{ab}}$ \\
\hline 10 & $71.7 \pm 17.2^{\text {cd }}$ & $32.7 \pm 4.6^{\mathrm{c}}$ & $30.7 \pm 10.4^{\mathrm{bc}}$ & $8.3 \pm 3.2^{\mathrm{bc}}$ \\
\hline 11 & $81.0 \pm 20.7^{\mathrm{cd}}$ & $35.7 \pm 5.5^{\mathrm{c}}$ & $38.0 \pm 15.1^{\mathrm{bc}}$ & $7.3 \pm 1.5^{\mathrm{abc}}$ \\
\hline 12 & $83.7 \pm 12.1^{\mathrm{cd}}$ & $26.7 \pm 3.2^{\mathrm{bc}}$ & $48.0 \pm 10.6^{\mathrm{c}}$ & $9.0 \pm 1.7^{\mathrm{bc}}$ \\
\hline 13 & $96.3 \pm 31.4^{\mathrm{d}}$ & $27.7 \pm 1.2^{\mathrm{bc}}$ & $54.3 \pm 21.7^{\mathrm{c}}$ & $14.3 \pm 9.6^{\mathrm{c}}$ \\
\hline 14 & $88.7 \pm 30.0^{\mathrm{cd}}$ & $29.0 \pm 9.2^{\mathrm{bc}}$ & $46.3 \pm 14.2^{\mathrm{c}}$ & $13.3 \pm 2.1^{\mathrm{c}}$ \\
\hline
\end{tabular}

Total 24 female B6CBAF1 mice were euthanized for retrieving preantral follicles.

Model effects of the treatment on the mean number of follicles retrieved, and the mean number of retrieved follicles at the stage of primary, early secondary and late secondary were $0.0005,0.0013,0.0012$ and 0.0052 , respectively.

${ }^{\mathrm{a}-\mathrm{d}}$ Different superscripts within the same parameter indicate significant difference among the treatments, $\mathrm{p}<0.05$.

of 7- to 14-day-old $(\mathrm{n}=24$; three in each age) was 10.7 , $36.3,53.7,71.7,81,83.7,96.3$ and 88.7 , respectively (Table 1). Significant $(\mathrm{p}=0.0005)$ increase in the retrieval number was detected from the 9-day-old, which was peaked at the 13-day-old. The number of primary follicles was gradually increased to 35.7 follicles up to 11-day-old, then reduced to 29 follicles at 14-day-old ( $\mathrm{p}=0.0013)$. The number of early (2.0 to 54.3 follicles) and late (0 to 14.3 follicles) secondary follicles were also increased continuously up to 13-day-old $(\mathrm{p}=0.0052)$.

Experiment 2: Growth of primary follicles and developmental competence of intrafollicular oocytes matured in primary follicles

As shown in Table 2, more primary follicles retrieved from 10-day-old or 11-day-old females maintained their normal morphological integrity at the end of primary culture than the follicles retrieved from 9 -day-old $(n=7$ in each age; $p=0.0086)$. Of those, number of the follicles developing into the early secondary follicle was significantly higher in the group of 11-day-old than in the others (39 vs. 13 to $29 \%$ ). Compared with the 10-day-old and 11-day-old, none of the 9-day-old retrieved follicles grew into the late secondary follicles at the end of primary culture. However, there was no significant difference in the number of primary follicles developing into the pseudoantral stage between the retrieval ages at the end of the secondary culture ( 21 to $30 \% ; \mathrm{p}=0.5222)$. No significant difference was also detected in the number of oocytes mucified between the retrieval ages ( 32 to $39 \%$; $=$ $0.5795)$.

Next, developmental competence of intrafollicular oocytes from primary follicle culture was monitored (Table $3)$. Average number of mature oocytes per animal was increased more than 3.3 times in the group of 11-day-old retrieval than in the group of 9-day-old retrieval ( 0.86 to

Table 2. Follicular growth during in-vitro culture of primary follicles retrieved from the mice ${ }^{\mathrm{a}}$ of different ages

\begin{tabular}{|c|c|c|c|c|c|c|c|}
\hline \multirow{2}{*}{$\begin{array}{l}\text { Ages at retrieval } \\
\text { (days) }\end{array}$} & \multicolumn{2}{|c|}{ No. $(\%)^{\mathrm{b}}$ of primary follicles } & \multicolumn{3}{|c|}{ Diameter $(\mu \mathrm{m})$ of normal follicles $\mathrm{s}^{\mathrm{c}}$} & \multicolumn{2}{|c|}{ No. $(\%)^{\mathrm{d}}$ of } \\
\hline & Cultured & $\begin{array}{c}\text { Normal } \\
\text { morphology }\end{array}$ & $75-99(\%)^{\mathrm{d}}$ & $100-125(\%)^{\mathrm{d}}$ & $<125(\%)^{\mathrm{d}}$ & $\begin{array}{l}\text { Pseudo-antral } \\
\text { follicles }\end{array}$ & $\begin{array}{l}\text { Oocytes } \\
\text { mucified }\end{array}$ \\
\hline 9 & 109 & $63(58)^{\mathrm{f}}$ & $49(45)$ & $14(13)^{\mathrm{f}}$ & $0(0)^{\mathrm{f}}$ & $14(21)$ & $20(32)$ \\
\hline 10 & 157 & $113(72)^{\mathrm{g}}$ & $60(38)$ & $45(29)^{\mathrm{g}}$ & $8(5)^{\mathrm{g}}$ & $34(30)$ & $44(39)$ \\
\hline 11 & 158 & $118(75)^{\mathrm{g}}$ & $53(34)$ & $61(39)^{\mathrm{h}}$ & $4(3)^{\mathrm{fg}}$ & $31(26)$ & $46(39)$ \\
\hline
\end{tabular}

Primary follicles were cultured by a two-step method.

Model effects of the treatment on the number of primary follicles with normal morphology, the diameter of normal follicles with 75-99 $\mu \mathrm{m}$, 100-124 $\mu \mathrm{m}$ and more than $125 \mu \mathrm{m}$, and the number of pseudoantral follicles and follicles mucified, which were indicated as p values, were $0.0086,0.1698$, less than 0.0001 and $0.046,0.5222$ and 0.5795 , respectively.

${ }^{a}$ Total 21 female F1 mice were euthanized and each treatment replicated 7 times.

${ }^{\mathrm{b}}$ Percentage of the number of primary follicles cultured.

${ }^{\mathrm{c}}$ Only primary follicles with normal morphology on day 4 of culture were transferred to the secondary culture medium and their sizes were measured at the end of culture.

${ }^{\mathrm{d}}$ Percentage of the number of follicles with normal morphology.

${ }^{\mathrm{e}}$ Percentage of the number of follicles mucified.

${ }^{\text {fgh }}$ Different superscripts within the same parameter indicate significant differences among the treatments, $\mathrm{p}<0.05$. 
Table 3. Maturation of intrafollicular oocytes by in-vitro culture of primary follicles retrieved from the mice of different ages

\begin{tabular}{lccccc}
\hline \multirow{2}{*}{$\begin{array}{c}\text { Ages at retrieval } \\
\text { (days) }\end{array}$} & \multirow{2}{*}{$\begin{array}{c}\text { No. of females } \\
\text { euthanized }\end{array}$} & $\begin{array}{c}\text { No. of primary follicles } \\
\text { with normal } \\
\text { morphology }\end{array}$ & $\begin{array}{c}\text { No. of intrafollicular oocytes } \\
\text { Developed to the germinal vesicle } \\
\text { breakdown stage }(\%)^{\mathrm{a}}\end{array}$ & Matured $(\%)^{\mathrm{a}}$ & $\begin{array}{c}\text { Mean no. of mature } \\
\text { oocytes per female }\end{array}$ \\
\hline 9 & 7 & 63 & $12(19)$ & $6(10)$ & $0.86^{\mathrm{b}}$ \\
10 & 7 & 113 & $19(17)$ & $13(12)$ & $1.86^{\mathrm{bc}}$ \\
11 & 7 & 118 & $30(25)$ & $20(17)$ & $2.86^{\mathrm{c}}$ \\
\hline
\end{tabular}

Primary follicles were cultured by a two-step method.

Model effects of the treatment on the number of intrafollicular oocytes developed to the germinal vesicle breakdown stage and matured, and mean number of mature oocytes per female, which were indicated as p values, were $0.2557,0.2389$ and 0.0616 , respectively.

${ }^{a}$ Percentage of the number of primary follicles with normal morphology.

${ }^{\text {bc }}$ Different superscripts within the same parameter indicate significant differences among the treatments, $\mathrm{p}<0.05$.

2.86; $\mathrm{p}=0.0616)$. However, the initiation of meiosis estimated by germinal vesicle breakdown (17 to $25 \%$; $\mathrm{p}=$ $0.2557)$ and the completion of meiotic maturation (10 to $17 \% ; \mathrm{p}=0.2389$ ) were similar between retrieval ages. Morphology of developing follicles and mature oocytes was depicted in Figure 1, which confirmed morphological normality in each stage of development.

\section{DISCUSSION}

The results of this study demonstrated that there is a remarkable difference in in vitro-growth of primary follicles retrieved from neonatal mice of different ages, which suggests a positive correlation between number of retrieved
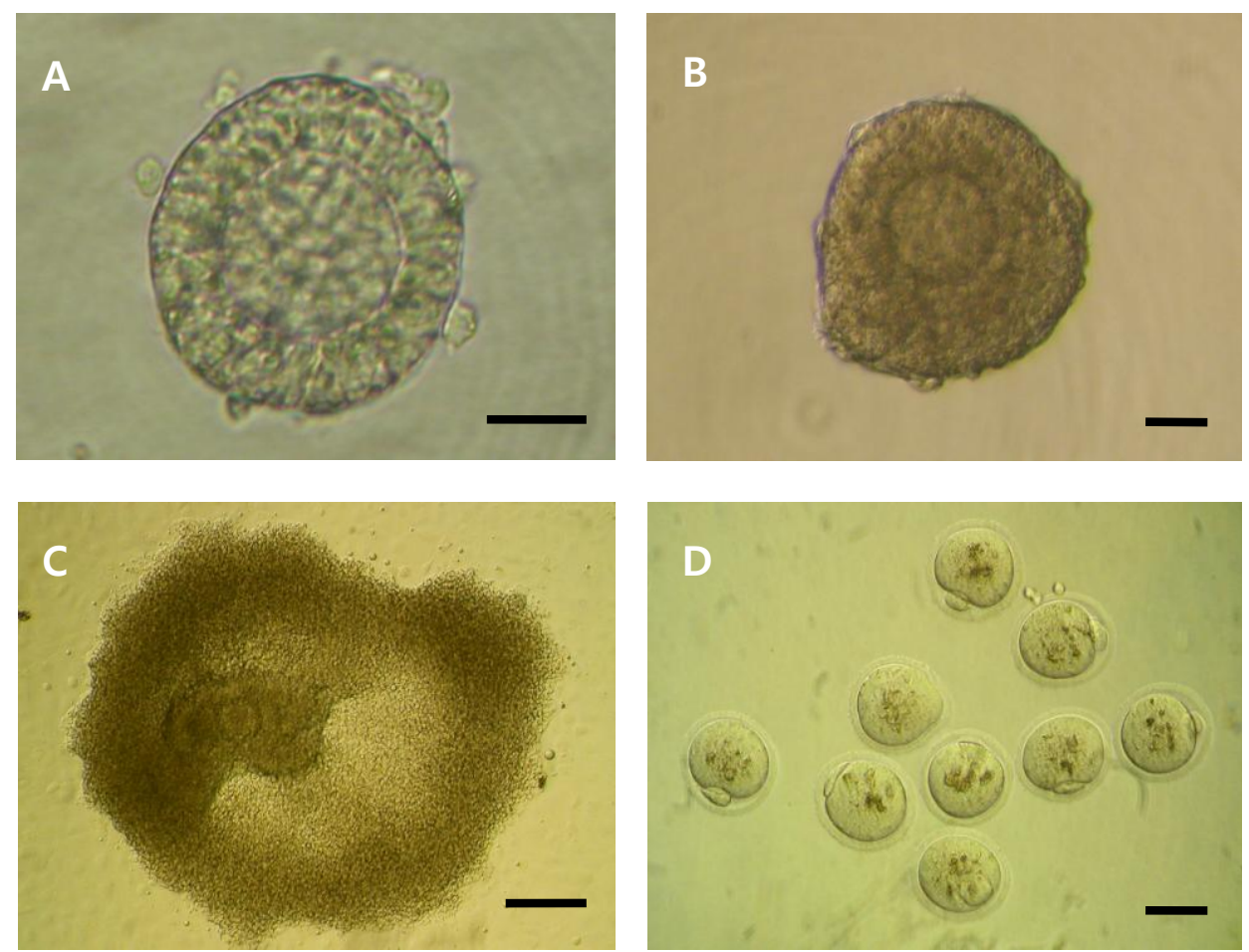

Figure 1. Morphology of primary follicles during in vitro culture and the follicle-derived oocytes. Primary follicle immediately after isolation (A), the cultured follicle (day 4) at the proliferative stage (B), the cultured follicles developing to the pseudoantral stage (C), mature (metaphase II stage) oocytes derived from in vitro-cultured primary follicles (D). (A and B: bar $=25 \mu \mathrm{m} ; \mathrm{C}$ to $\mathrm{D}: \mathrm{bar}=50 \mu \mathrm{m})$. 
for the derivation of mature oocytes from primary follicles except for the experiment of Lenie et al. (2004). The report showed that primary follicles were retrieved 8- to 14 dayold females and yielded mature oocytes with a two step follicle culture system, which consists of a 6 days of primary and a 12 days of secondary culture, respectively. Our study further suggests the optimal time for the retrieval of primary follicles from neonatal female was 11-day-old and the total culture time for the two-step system was 11 days including a 4 days of primary and a 7 days of secondary culture. This discrepancy may result from the differences of medium composition and miscellaneous culture regimes. Accordingly, primary follicles of 9- to 11day-old that used in this study may require different microenvironment for optimal growth in vitro, compared with the previous report with the use of 8- to 14-day-old primary follicles.

It is hypothesized that primary follicle may be more sensitive to culture environment than secondary follicle of advanced stage. The fact that primary follicle is encompassed with only single or several layers of primitive granulosa cell layer may induce high susceptibility to exogenous environment. In other words, the culture outcome of primary follicle may be more influenced by cell-to-cell interaction or exogenous environment during in vitro-manipulation than that of secondary follicles. Accordingly, the optimization of manipulation protocols such as retrieval time, miscellaneous change of medium composition and culture duration definitely contributes to enhancing the capacity of primary follicle to derive developmentally competent oocytes in vitro.

There are various paracrine factors involved in regulating follicle growth (McGrath et al., 1995; Dube et al., 1998; Gilchrist et al., 2001; Markstrom et al., 2002). Moreover, it has been suggested that the growth and maturation of intrafollicular oocytes in preantral follicles are significantly influenced by cell to cell interaction between oocytes and surrounding follicular cells (granulosa and theca cells) through paracrine methods (Eppig et al., 2002). For instance, paracrine factors secreted by oocytes (i.e. growth differentiation factor 9 and bone morphogenic protein 15) stimulate growth of granulosa cells and expression of folliculogenesis-related genes (Dube et al., 1998; Sadeu et al., 2008) and c-kit ligand stimulates the growth of intrafollicular oocytes retrieved from neonatal mice (Packer et al., 1994; Driancourt et al., 2000). Indeed, maturational status of follicular cells is one of the critical factors for successful culture of primary follicles. In this study, we used culture media supplemented $200 \mathrm{mIU}$ FSH during culture period and observed a great expansion of follicle cells with changing their morphology, which might imply functional acquisition of follicular cells by in vitroculture of primary follicles. Further study is needed for refining some factors which work more critically in growth of primary follicles than that of secondary follicles.

In conclusion, we provide further information on optimizing of primary follicle culture protocol in terms of follicle retrieval. Since a significant difference in primary follicle retrieval and the number of mature oocyte per the follicle donor is observed during neonatal period, selection of retrieval time is definitely necessary to increase the efficiency. Therefore, the follicle retrieval during mid neonatal (approximately less than 3 weeks after birth) period can be recommended for the establishment of in vitro primary follicle culture system. This suggested guideline will contribute to establishing a new ART strategy, as well as to developing novel regenerative medicine employing embryo-derived, pluripotent cells. We are now confirming the feasibility of oocytes derived from in vitro-cultured, primary follicles for generating viable embryos and for establishing stem cell-like cells.

\section{ACKNOWLEDGMENTS}

The submitted materials were partially presented in platform presentation session of 2007 annual meeting of society for the study of reproduction held in San Antonio, USA. This research was supported by a grant (SC-5160) from the Stem Cell Research Center of the 21st Century Frontier Research program funded by the Ministry of Education, Science and Technology (MEST) of the Republic of Korea. The authors also acknowledge a World Class University (WCU) program (R-31-10056), the National Research Foundation of Korea funded by the MEST.

\section{REFERENCES}

Cortvrindt, R. G., J. Smitz and A. C. Van Steirteghem. 1996. Invitro maturation, fertilization and embryo development of immature oocytes from early preantral follicles from prepubertal mice in a simplified culture system. Hum. Reprod. 12:759-768.

Driancourt, M. A., K. Reynaud, R. Cortvrindt and J. Smitz. 2000. Roles of Kit and Kit ligand in ovarian function. Rev. Reprod. 5:143-152.

Dube, J. L., P. Wang, J. Elvin, K. M. Lyons, A. J. Celeste and M. M. Matzuk. 1998. The bone morphogenetic protein 15 gene is $\mathrm{X}$-linked and expressed in oocytes. Mol. Endocrinol. 12:18091817.

Eppig, J. J., K. Wigglesworth and F. L. Pendola. 2002. The mammalian oocytes orchestrates the rate of ovarian follicular development. Proc. Natl. Acad. Sci. USA. 99:2890-2894.

Gilchrist, R. B., L. J. Ritter and D. T. Armstrong. 2001. Mouse oocytes mitogenic activity is developmentally coordinated throughout folliculogenesis and meiotic maturation. Dev. Biol. 240:289-298

Gong, S. P., E. J. Lee, S. T. Lee, H. Kim, S. H. Lee, H. J. Han 
and J. M. Lim. 2008. Improved establishment of autologous stem cells derived from preantral follicle culture and oocyte parthenogenesis. Stem Cells Dev. 17:695-712.

Gong, S. P., H. Kim, E. J. Lee, S. T. Lee, S. Moon, H. J. Lee and J. M. Lim. 2009. Change in gene expression of mouse embryonic stem cells derived from parthenogenetic activation. Hum. Reprod. 24:805-814.

Kim, I. W., S. P. Gong, C. R. Yoo, J. H. Choi, D. Y. Kim and J. M. Lim. 2009. Derivation of developmentally competent oocytes by the culture of preantral follicles retrieved from adult ovaries: maturation, blastocyst formation, and embryonic stem cell transformation. Fertil. Steril. 92:1716-1724.

Lee, S. T., M. H. Choi, S. P. Gong, J. Y. Han and J. M. Lim. 2007. Establishment of a basic method for manipulating preantral follicles: effects of retrieval method on in vitro growth of preantral follicles and intrafollicular oocytes. Zygote 15:109116.

Lee, S. T., M. H. Choi, E. J. Lee, S. P. Gong, M. Jang, S. H. Park, H. Jee, D. Y. Kim, J. Y. Han and J. M. Lim. 2008. Establishment of autologous embryonic stem cells derived from preantral follicle culture and oocyte parthenogenesis. Fertil. Steril. 90:1910-1920.

Lenie, S., R. Cortvrindt, T. Adriaenssens and J. Smitz. 2004. A reproducible two step culture system for isolated primary mouse ovarian follicles as single functional units. Biol. Reprod. 71:1730-1738.

Markstrom, E., E. C. Svensson, R. Shao, B. Svanberg and H. Biling. 2002. Survival factors regulating ovarian apoptosisdependence on follicle differentiation. Reproduction 123:23-30.
McGrath, S. A., A. F. Esquela and S. J. Lee. 1995. Oocyte-specific expression of growth/differentiation factor-9. Mol. Endocrinol. 9:131-136

Nayudu, P. L. and S. M. Osborn. 1992. Factors influencing the rate of preantral and antral growth of mouse ovarian follicles in vitro. J. Reprod. Fertil. 95:349-362.

O’Brien, M. J., J. K. Pendola and J. J. Eppig. 2003. A revised protocol for in vitro development of mouse oocytes from primordial follicles dramatically improves their developmental competence. Biol. Reprod. 68:1682-1686.

Packer, A. I., Y. C. Hsu, P. Besmer and R. F. Bachvarova. 1994. The ligand of the c-kit receptor promotes oocyte growth. Dev. Biol. 161:194-205.

Sadeu, J. C., T. Adrianssens and J. Smitz. 2008. Expression of growth differentiation factor 9 , bone morphogenic protein 15 , and anti Mullerian hormone in cultured mouse primary follicles. Reproduction 136:195-203.

Sirotkin, A. V. 1996. Direct action of growth hormone on bovine ovarian cells: effects on estradiol, oxytocin, vasopressin release by granulosa cells and on oocyte maturation and cleavage in vitro. Ann. Endocinol. 57:219-224.

Smitz, J. E. and R. G. Cortvrindt. 2002. The earlist stages of folliculogenesis in vitro. Reproduction 123:185-202.

Tajima, K., M. Orisaka, H. Yata, K. Goto, K. Hosokawa and F. Kotsuji. 2006. Role of granulosa and theca cell interactions in ovarian follicular maturation. Microsc. Res. Tech. 69:450458.

Zeuner, A., K. Muller, K. Requszynski and K. Jewqenow. 2003. Apoptosis within bovine follicular cells and its effect on oocyte development during in vitro maturation. Theriogenology 59:1412-1433. 